\title{
Cefalea rinogénica ¿mito o realidad?: \\ Displasia fibrosa de cornete medio como causa de algia facial
}

\author{
Rhinogenic headache ¿myth or reality?: \\ Middle turbinate fibrous displasia, as a cause of facial pain
}

Lara Fernández R'ㄹ, Paul Boettiger B' ${ }^{1}$, Karol Baksai E², Paul H. Délano $\mathbf{R}^{1,3}$.

\begin{abstract}
RESUMEN
La cefalea es uno de los motivos de consulta más frecuentes en medicina y se clasifica como primaria en cefaleas tensionales o migrañas y secundaria a tumores, infecciones u otras causas. Dentro del grupo de cefaleas o algias faciales secundarias se plantea la existencia del cuadro clínico de cefalea rinogénica generada por puntos de contacto de la mucosa de la cavidad nasal. En este artículo se presenta un caso de una paciente que posterior al fracaso del tratamiento farmacológico para migraña, consultó por algia facial persistente. Al realizar un examen clínico y con tomografía computarizada de las cavidades perinasales, se diagnosticó una cefalea rinogénica por contacto de mucosa secundaria a una displasia fibrosa del cornete medio. La resección quirúrgica del punto de contacto a través de una turbinectomía media realizada por cirugía endoscópica nasal abolió totalmente el dolor facial. Este caso recalca la utilidad y necesidad del estudio de las cavidades perinasales en aquellos pacientes que presentan cefalea 0 algia facial.
\end{abstract}

Palabras clave: Cefalea, cornete medio, displasia fibrosa, cefalea rinogénica.

\begin{abstract}
Headache is one of the most frequent symptoms in medicine that can be classified as primary, like tensional headache and migraine or secondary as in tumors, infections, or other causes. Rhinogenic headache is proposed as a clinical entity secondary to mucosal contact points within the nasal cavity. In this article we present a case of a patient that after a pharmacological treatment failure for migraine, consulted for sustained facial pain. After clinical examination and a paranasal sinus computed tomography, a rhinogenic headache secondary to middle turbinate fibrous dysplasia was diagnosed. Endoscopic surgical extirpation of contact mucosal points by a medial turbinectomy produced complete abolition of facial pain. This case illustrates the need and utility of studying paranasal sinus in those patients with headache or facial pain.
\end{abstract}

Key words: Headache, middle turbinate, fibrous dysplasia, rhinogenic headache.

\footnotetext{
${ }^{1}$ Servicio de Otorrinolaringología, Hospital Clínico Universidad de Chile, Santiago, Chile.

${ }^{2}$ Servicio de Anatomía Patológica, Hospital Clínico Universidad de Chile, Santiago, Chile.

${ }^{3}$ Programa de Fisiología y Biofísica, ICBM, Facultad de Medicina, Universidad de Chile, Santiago, Chile.
} 


\section{INTRODUCCIÓN}

La cefalea es uno de los motivos de consulta más frecuentes en medicina. Se estima que cerca del $50 \%$ a $60 \%$ de la población general padece de este síntoma en algún momento de su vida ${ }^{1,2}$. La sociedad internacional de cefalea clasifica a éstas en primarias y secundarias, incluyendo en el primer grupo a la cefalea tensional, migraña y cefaleas en racim $0^{3}$. Las cefaleas secundarias agrupan a diversas patologías incluyendo tumores cerebrales, traumas encéfalocraneanos, infecciones y algias faciales de origen craneal, ocular o nasal entre otras. Específicamente, se clasifica a la cefalea rinogénica en la categoría 11.5, la que corresponde al dolor del macizo facial asociado a un cuadro de rinosinusitis aguda ${ }^{3}$.

La existencia del cuadro clínico de cefalea rinogénica producida por un punto de contacto entre sitios de la mucosa de la cavidad nasal es controversial ${ }^{4,5}$. Este tipo de cefalea, ha sido clasificada transitoriamente según la sociedad internacional de cefalea en el punto 11.5.1 y sería producida por desviaciones del tabique nasal, hipertrofia de cornetes 0 tumores nasales ${ }^{3-5}$. Para el diagnóstico de certeza de este tipo de cefalea se debe confirmar la presencia de puntos de contactos mucosos a través de endoscopía nasal o imágenes como la tomografía computarizada, y excluyendo causas neurológicas, oftalmológicas y dentales de cefalea. Un criterio diagnóstico aceptado es la abolición del dolor a los 5 minutos de la aplicación de un anestésico tópico en el sitio de contacto mucoso $0^{5,6}$.

Se ha propuesto la extirpación quirúrgica del punto de contacto mucoso nasal como tratamiento de este tipo cefaleas rinogénicas. Si bien existen reportes clínicos de pacientes con este tipo de cefalea en que el manejo quirúrgico ha sido curativo 0 al menos ha disminuido la intensidad del dolor, aún es controversial indicar cirugía a pacientes con cefalea rinogénica ${ }^{7-12}$.

En este artículo presentamos un caso clínico de una paciente con cefalea refractaria a tratamiento farmacológico en la que se planteó el diagnóstico de cefalea rinogénica secundaria a displasia fibrosa de cornete medio.

\section{CASO CLíNICO}

Paciente de sexo femenino de 43 años que consulta al servicio de otorrinolaringología por cefalea frontal y algia periorbitaria diaria de 10 años de evolución. La paciente además reporta obstrucción nasal bilateral e hiposmia. Tiene evaluación neurológica y tomografía computarizada de cerebro normal. En neurología se indicó tratamiento con carbamazepina por posible neuralgia del trigémino y luego con naratriptan por probable migraña sin aura, ambos tratamientos sin resultados. La paciente refiere tener un dolor intenso y discapacitante, 9 de 10 en escala visuo-análoga.

Al examen rinoscópico se observa un aumento de volumen del cornete medio derecho sin alteraciones de la mucosa nasal. Se solicita tomografía computarizada de cavidades perinasales que muestra imagen tipo vidrio esmerilado del cornete medio derecho sugerente de displasia fibrosa que contacta al tabique nasal (Figura 1).

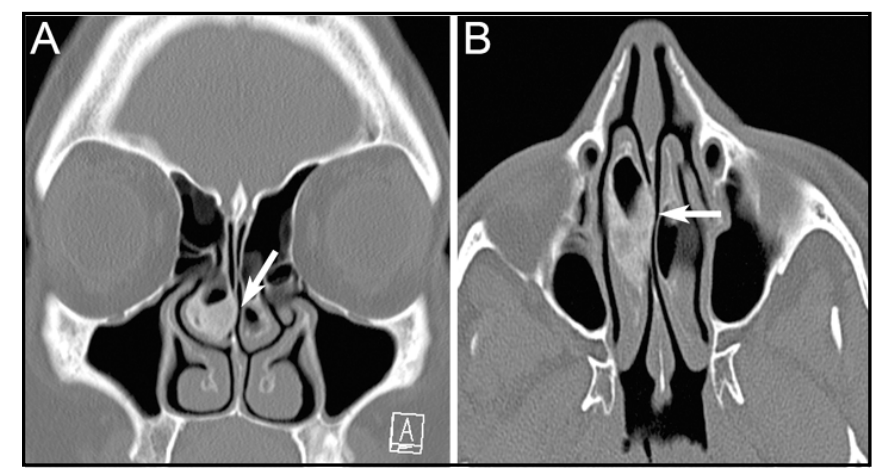

Figura 1. Tomografía computarizada de cavidades perinasales. (A) imagen coronal: Se observa lesión tipo vidrio esmerilado que se ubica en forma aislada en el cornete medio derecho. La flecha blanca muestra el punto de contacto entre la mucosa del cornete medio derecho y el tabique nasal. (B) Imagen axial: Se observa la misma lesión y punto de contacto con tabique nasal. Note que los cornetes medios se encuentran neumatizados (concha bullosa). 
Se diagnostica una cefalea rinogénica secundaria a displasia osteofibrosa del cornete medio y se realiza turbinectomía media derecha a través de cirugía endoscópica nasal. El cornete medio se encuentra aumentado de tamaño $(4,0 \times 1,5 \times 1,2 \mathrm{~cm})$ y consistencia. La biopsia de la lesión muestra estroma fibroso compuesto de células fusadas sin atipias y trabéculas óseas inmaduras de forma y tamaño irregular, concordante con displasia fibrosa (Figura 2).

Posterior a la intervención quirúrgica, la paciente evoluciona en buenas condiciones sin complicaciones. La cefalea y el dolor facial ceden progresivamente y desaparecen de forma definitiva 48 horas después de la cirugía, con un valor de 0 de 10 puntos en escala visuo-análoga de dolor. La paciente ha sido controlada a los 3 y 6 meses posoperatorios, encontrándose asintomática.

\section{DISCUSIÓN}

La displasia fibrosa es un desorden displástico del hueso, que en la región craneofacial, afecta predominantemente al seno maxilar y la región fronto-orbitaria, siendo infrecuente su localización en cornete medio. Estas lesiones generan aumento de volumen doloroso o deformidad facial pero usualmente se diagnostican en forma incidental mediante imágenes ${ }^{13,14}$. Existen 2 variantes ${ }^{15}$, el fibroma osificante, agresivo y recurrente, y la displasia fibrosa, presentándose esta última de forma monostótica en cerca del $70 \%$ de los casos, poliostótica en el 20\%-30\% de los casos y como síndrome de McCune-Albright cuando se presenta en forma poliostótica y se asocia a pubertad precoz, hiperpigmentación (máculas tipo "café con leche") y secreción aumentada de diversas hormonas ${ }^{16,17}$.

Habitualmente el manejo de la displasia fibrosa no es quirúrgico, excepto cuando se produce algún tipo de complicación secundaria a la compresión ósea. En el caso presentado, dado la cefalea persistente y refractaria a tratamiento farmacológico y la presencia de un tumor nasal que producía contacto entre mucosas de la cavidad nasal, se planteó el diagnóstico de cefalea rinogénica y se extirpó el tumor con evolución favorable y alivio del dolor para la paciente.

Dentro del diagnóstico diferencial se debe considerar a la migraña sin aura, e incluso algunos autores sugieren que la cefalea rinogénica, más que un diagnóstico aislado, correspondería a una migraña sin aura que se gatilla por el contacto mucoso en la cavidad nasal ${ }^{3,18}$. Se debe considerar que la migraña sin aura y la cefalea 0 algia facial rinogénica pueden ser cuadros clínicos que se presentan en forma simultánea y muchas veces los pacientes son derivados desde neurología a otorrinolaringología o viceversa sin tener un diagnóstico claro. Es importante destacar que independiente de si corresponde a una migraña o exclusivamente a una cefalea de origen nasal, en ambos casos la resección quirúrgica del punto de contacto mucoso sería útil como tratamiento ${ }^{19,20}$.

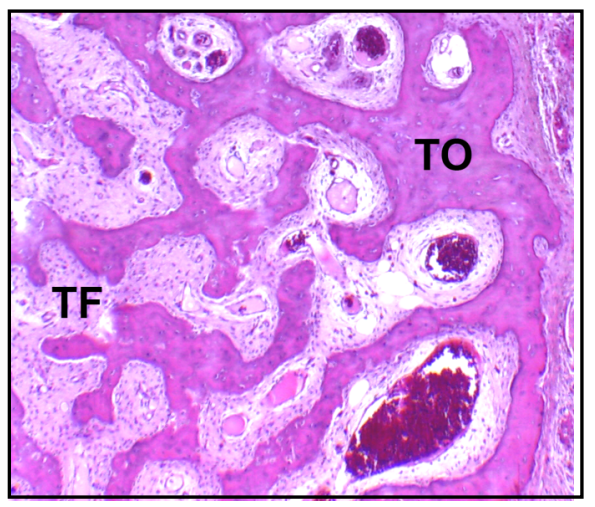

Figura 2. Histología de cornete medio compatible con displasia fibrosa. Tinción hematoxilina-eosina, aumento 40X. Se observa estroma fibroso (TF) sin atipias y trabéculas óseas inmaduras (T0) curvilíneas y anastomosantes entre sí, con ausencia de ribete osteoblástico, compatible con displasia fibrosa (foto gentileza de Dr. Facundo Las Heras, Servicio Anatomía Patológica Hospital Clínico Universidad de Chile). 


\section{CONCLUSIONES}

La cefalea es un motivo de consulta habitual en medicina siendo la migraña y la cefalea tensional los diagnósticos más frecuentes de realizar. El caso presentado en este artículo apoya la existencia de la cefalea rinogénica como una entidad diagnóstica. Es importante tener en mente el diagnóstico de cefalea rinogénica secundaria a puntos de contacto mucoso endonasal en aquellos casos de algia facial o migraña sin aura con algia periocular. En estos casos se debe solicitar una tomografía computarizada de cavidades perinasales y un estudio endoscópico nasal.

\section{BIBLIOGRAFÍA}

1. Pascual J, Colás R, Castillo J. Epidemiology of chronic daily headache. Curr Pain Headache Rep 2001; 5(6): 529-36.

2. Stovner LJ, Andree C. Prevalence of headache in Europe: a review for the Eurolight project. J Headache Pain 2010; 11(4): 289-99. Epub 2010 May 16.

3. Headache Classification Subcommittee of the International Headache Society. The International Classification of Headache Disorders: $2^{\text {nd }}$ edition. Cephalalgia 2004; 24 (Suppl 1): 9-160.

4. Blumenthal HJ. Headaches and sinus disease. Headache. 2001; 41: 883-88.

5. Cady RK, Dodick DW, Levine HL et al. Sinus Headache: A Neurology, Otolaryngology, Allergy, and Primary Care Consensus on Diagnosis and Treatment. Mayo Clin Proc 2005; 80(7): 908-16.

6. Roozbahany NA, Naski S. Auris Nasus Larynx. Nasal and paranasal sinus anatomical variations in patients with rhinogenic contact point headache. En Prensa, 2012.

7. Tosun F, Gerek M, Ozkaptan Y. Nasal surgery for contact point headaches. Headache 2000; 40: 237-40.

8. Mohebil A, Memari F, MoheBbi S. Endonasal endoscopic management of contact point headache and diagnostic criteria. Headache 2010; 50(2): 242-8.
9. Behin F, Behin B, Behin D, Baredes S. Surgical management of contact point headaches. Headache 2005; 45(3): 204-10.

10. Welge-Luessen A, Hauser R, Schmid N, Kappos L, Probst R. Endonasal surgery for contact point headaches: a 10-year Iongitudinal study. Laryngoscope 2003; 113(12): 2151-6.

11. Abu-Samra M, Gawad OA, Agha M. The outcomes for nasal contact point surgeries in patients with unsatisfactory response to chronic daily headache medications Eur Arch Otorhinolaryngol 2011; 268: 1299-304.

12. Harley DH, Powitzky ES, Duncavage J . Clinical outcomes for the surgical treatment of sinonasal headache. Otolaryngol Head Neck Surg 2003; 129: 217-21.

13. Alba JR, Armengot M, Pérez CA, et al. An exceptional form of craneo-facial fibrous dysplasia: fibrous dysplasia of middle turbinate. Acta Otorrinolaringol Esp 2002; 53: 291-4.

14. Ozcan KM, Akdogan O, Gedikl Y, Ozcanl, Dere H, UnaL T. Fibrous dysplasia of inferior turbinate, middle turbinate, and frontal sinus. B-ENT2007;3(1): 35-8.

15. Galvan 0, Gasnner EM, Neher A, Gunkel AR. Fibroosseous lesion of the middle turbinate: ossifying fibroma or fibrous dysplasia? J Laryngol Otol 2007; 121(12): 1201-3.

16. Dumitrescu CE, Collins MT. McCune-Albright syndrome. Orphanet J Rare Dis 2008; 3: 12.

17. LaBagnara J, Yuppa F, Klein MH. Fibrous dysplasia of the middle turbinate with primary hyperparathyroidism. Ear Nose Throat J 1998; 77(7): 552-3.

18. Clerico DM. Pneumatized superior turbinate as a cause of referred migraine headache. Laryngoscope 1996; 106: 874-9.

19. Behin F, Behin B, Bigal ME, Lipton RB. Surgical treatment of patients with refractory migraine headaches and intranasal contact points. Cephalalgia 2005; 25(6): 439-43.

20. Behin F, Lipton RB, Bigal M. Migraine and intranasal contact point headache: is there any connection? Curr Pain Headache Rep 2006; 10(4): 312-5.

Dirección: Dr. Paul H. Délano R.

Santos Dumont 999, Independencia, Santiago

E mail: phdelano@gmail.com 